\title{
Tunable three-dimensional graphene assembly architectures through controlled diffusion of aqueous solution from a micro-droplet
}

\author{
Jin Gook Bae ${ }^{1}$, Minsu Park ${ }^{1}$, Do Hyun Kim ${ }^{1}$, Eun Yeol Lee ${ }^{2}$, Woo-Sik Kim² and Tae Seok Seo ${ }^{1,2}$
}

The two-dimensional (2D) graphene sheets show superior electrical, thermal and mechanical properties. The three-dimensional (3D) graphene assemblies have also recently garnered great attention because of their high surface area, free-standing configuration and facile fabrication of graphene composites with nanomaterials in practical applications. Herein, we demonstrate the synthesis of a variety of 3D graphene assemblies including spheres, twiddles and hemispheres using micro-droplet reactors. Such 3D reduced graphene oxide ( $\mathrm{rGO}$ ) structures can be manipulated by controlling the aggregation pattern of the GO sheet inside the micro-droplets. The aggregation pattern depends on the diffusion rate of the aqueous solution in the droplet. The pattern was simply tuned by the amount of oil phase in the droplet during evaporation. In addition, micro-porous structures were manufactured by incorporating silica beads in the rGO microparticles, followed by a wet etching process. The local plasmonic properties of the 3D porous hemispherical rGO were investigated by electron energy-loss spectroscopy mapping.

NPG Asia Materials (2016) 8, e329; doi:10.1038/am.2016.176; published online 18 November 2016

\section{INTRODUCTION}

The superlative properties of graphene such as exceptional carrier mobility, thermal conductivity and mechanical strength have been demonstrated in a variety of research fields. ${ }^{1-3}$ Despite the great potential of graphene as a substitution for state-of-the-art materials, the difficulty of the fabrication processes for graphene, the lack of reliable techniques for integrating graphene into devices and their tendency of aggregation among graphene sheets remain challenges to overcome. ${ }^{4-8}$ Contrary to the two-dimensional (2D) graphene sheet, the assembly of the $2 \mathrm{D}$ graphene in a controllable way results in unique three-dimensional (3D) graphene materials. The 3D graphene structures show several advantages, such as aggregation resistance, high surface area, easy handling due to free-standing configuration and facile composition of graphene with nanomaterials, while the excellent electronic and mechanical properties were retained. ${ }^{7,9-12}$

The most popular method to change $2 \mathrm{D}$ graphene to a $3 \mathrm{D}$ graphene assembly is to use graphene oxide (GO) as an intermediate that can be produced on a large scale and retains high dispersibility in water owing to the oxygenous functional groups. Once the 3D GO structure was fabricated, the chemical or thermal reduction reaction could return the GO into rGO (reduced graphene oxide) whose atomic structure is similar to graphene with some defects. ${ }^{9-11,13-16}$ In this context, the fabrication of GO films and crumpled rGO microparticles has been reported. In the case of the GO films, gas bubbles or versatile organic solvents transferred the $\mathrm{GO}$ in a bulk solution to the liquid-air interface for assembly. ${ }^{17-21}$ Although the synthesis of a large-scale GO film was possible, the control of the film thickness and mechanical strength was not satisfactory. An aerosol system was employed for generating nano-sized droplet reactors that contained the GO solution and after thermal treatment, crumpled rGO particles were produced..$^{9,11,22}$ Despite the advantage of the continuous production process, the size distribution of the resultant rGO was poor and the rGO shape was limited to spheres. Therefore, a novel synthetic GO assembly system would be required to produce versatile 3D rGO with control of the morphology and size.

To this end, we developed a microfluidic droplet system combined with a controllable diffusion setup to fabricate spherical, twiddle and hollow hemispherical rGO microparticles. The microfluidic droplet system provides uniform droplets as a reactor, and the contents such as the GO concentration and $\mathrm{pH}$ value can be easily adjusted to tune the size and thickness of the 3D rGO. ${ }^{10,23-25}$ In addition, we hypothesized that the GO assembly inside the droplets can be manipulated by the diffusion rate of the aqueous solution of droplets producing unique 3D rGO structures. Furthermore, the silica bead incorporation into the $3 \mathrm{D}$ rGO enables us to synthesize porous rGO materials that reveal local surface and bulk plasmonic properties of graphene.

\section{MATERIALS AND METHODS}

Chip fabrication and operation

The microfluidic droplet chip was fabricated to produce water-in-oil droplets by conventional soft lithography with a channel depth and width of 100 and $75 \mu \mathrm{m}$, respectively. The polydimethylsiloxane chip, which was designed with one inlet for injecting a continuous oil phase, two inlets for introducing an

\footnotetext{
${ }^{1}$ Department of Chemical and Biomolecular Engineering, Korea Advanced Institute of Science and Technology (KAIST), Daejeon, Republic of Korea and ${ }^{2}$ Department of Chemical Engineering, College of Engineering, Kyung Hee University, Gyeonggi-do, Republic of Korea

Correspondence: Professor TS Seo, Department of Chemical Engineering, College of Engineering, Kyung Hee University, 1 Seochon-dong, Giheung-gu, Yongin-si, Gyeonggi-do 17140, Republic of Korea.

E-mail: seots@kaist.ac.kr

Received 27 May 2016; revised 12 September 2016; accepted 16 September 2016
} 
aqueous solution, a serpentine passive mixing microchannel and an outlet, was bonded with a glass wafer after ozone treatment (Figure 1a). Symmetrical injection channels and precise flow control ensured identical composition inside the resultant droplets.

The droplet chip was operated by a syringe pump with a flow rate of $20 \mu \mathrm{min}^{-1}$ for a continuous phase (hexadecane with $2 \mathrm{wt} \%$ span 80 (Sigma-Aldrich, St Louis, MO, USA)) and $2 \mu \mathrm{min}^{-1}$ for a dispersed phase that consisted of an aqueous GO solution, $\mathrm{pH}$ controlled deionized water and a silica-bead solution. The diameter of the nano-sized GO was $90 \pm 15 \mathrm{~nm}$ (Graphene Supermarket, Calverton, New York, USA), and the diameters of the silica beads were 1.0, 0.5 and $0.15 \mu \mathrm{m}$ (Sigma-Aldrich). In one dispersedphase inlet, the aqueous GO was introduced at varying concentrations, and in the other inlet, $\mathrm{pH}$ controlled water with or without a silica-bead solution was injected. Micro-droplets (diameter $76 \mu \mathrm{m}$ ) were formed at the flow-focusing zone and were collected on the square silicon wafer $(1.5 \mathrm{~cm} \times 1.5 \mathrm{~cm})$ that was previously coated by hexamethyldisilazane for $20 \mathrm{~min}$ (Figure 1b). The collected droplets settled down compactly on the wafer because the density of the droplets $\left(\rho_{\text {water }}=1.00 \mathrm{~g} \mathrm{~cm}^{-3}\right)$ was higher than that of the oil $\left(\rho_{\text {hexadecane }}=0.77 \mathrm{~g} \mathrm{~cm}^{-3}\right)$. The hydrophobic coating by hexamethyldisilazane enabled the droplets to be detached from the wafer surface. By measuring the collection time, a certain amount of droplets and oil could be gathered on the silicon wafer.

\section{Thermal annealing process for GO assembly and treatment of the} resultant $3 \mathrm{D}$ rGO particulates

The silicon wafers that collected droplets with a background of an oil phase were loaded into a tube furnace (Figure $1 \mathrm{~b}$ ). The furnace was heated up to $900{ }^{\circ} \mathrm{C}$ with a ramp rate of $3.75^{\circ} \mathrm{C} \mathrm{min}-1$, and maintained at $900{ }^{\circ} \mathrm{C}$ for $2 \mathrm{~h}$ under argon gas with $10 \%$ hydrogen gas $\left(100 \mathrm{cc} \mathrm{min}^{-1}\right)$. During the thermal

a
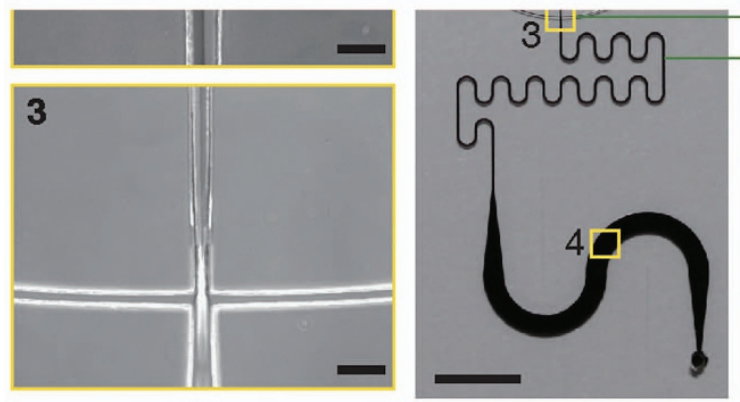

b

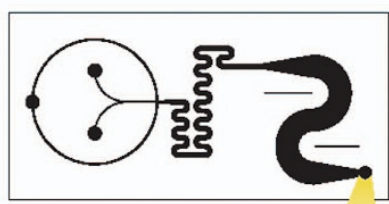

HMDS treated Si wafer

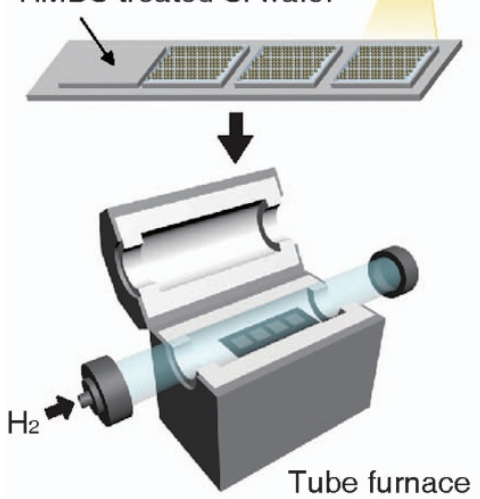

C

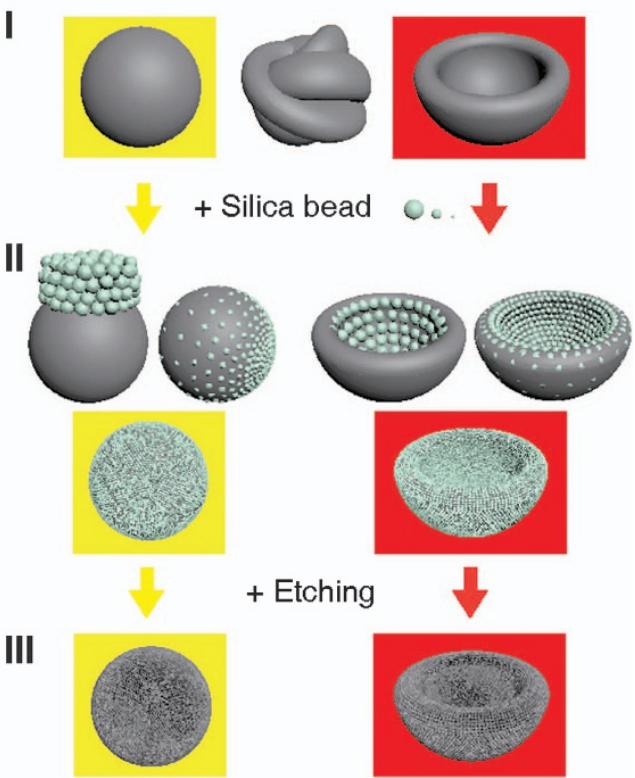

Figure 1 The process for fabricating diverse three-dimensional (3D) reduced graphene oxide (rGO) structures using a microfluidic droplet chip. (a, 1) The microfluidic droplet chip. (a, 2-4) Enlarged images of the merging point of the two aqueous solution inlets, the flow-focusing zone and the generated droplets. (b) Schematic illustration of the thermal treatment of the collected droplets on the hexamethyldisilazane (HMDS)-coated silicon wafer in the tube furnace. (c) A variety of morphologies of rGOs and their derivatives. (c, I) Spherical, twiddle and hollow hemispherical shape. (c, II) Spherical and hollow hemispherical rGO decorated with $\mathrm{SiO}_{2}$ beads. (c, III) Porous spherical and hollow hemispherical structure by etching silica beads in the rGO-SiO 2 composites. Scale bar (a, 1): $5 \mathrm{~mm}$ and (a, 2-4): $0.1 \mathrm{~mm}$. 
annealing process, the aqueous solution in the droplets was evaporated. Simultaneously, GOs were reduced to rGO. After evaporation, the 3D rGO microparticulates were detached from the wafer by ultrasonication in water for $20 \mathrm{~min}$. The GO assembly behavior could be manipulated by controlling the diffusion rate of the aqueous solution in the furnace, so that various 3D graphene particulates such as spheres, twiddles and hollow hemispheres could be formed (Figure 1c, I). In addition, the composites decorated with silica beads (Figure 1c, II) and the corresponding porous 3D rGO could be produced (Figure 1c, III). To generate pores in the 3D rGO, the collected $\mathrm{rGO}-\mathrm{SiO}_{2}$ composite was wet-etched by mixing in a $5 \%$ hydrofluoric acid solution for $\sim 15 \mathrm{~min}$. The composite microparticles were washed with deionized water at least three times with centrifugation to obtain the porous rGO structure.

\section{Images of the rGO microparticles}

The morphology of the microparticles was investigated by scanning electron microscopy, and the internal structure and the cross-section images were obtained by cutting the microparticulates by using a focused ion beam (Helios Nanolab 450 F1, FEI company, Hillsboro, OR, USA) followed by scanning electron microscopy imaging.

\section{Scanning transmission electron microscopy-electron energy loss spectroscopy (STEM-EELS) imaging experiments}

Transmission electron microscopy (TEM) and high-angle annular dark-fieldSTEM analysis was performed using a FEI Titan cubed G2 60-300 microscope operating at $80 \mathrm{kV}$. The microscope was equipped with a monochromator and a Gatan Quantum 965 EEL spectrometer (Gatan, Pleasanton, CA, USA). The spatial and energy resolution of the microscope in a monochromated STEM mode was 0.3 and $0.2 \mathrm{eV}$. An aperture size of $50 \mu \mathrm{m}$ and a camera length of $30 \mathrm{~mm}$ were used during EELS acquisition. This condition was equivalent to a convergence semiangle of $15.3 \mathrm{mrad}$ and a collection semiangle of $26.06 \mathrm{mrad}$. The EELS mapping was acquired with a spatial step of $0.5 \mathrm{~nm}$ over $2 \mathrm{D}$ regions $(94 \times 94$ pixels). The intensity of the EELS maps was coded in a color scale. a

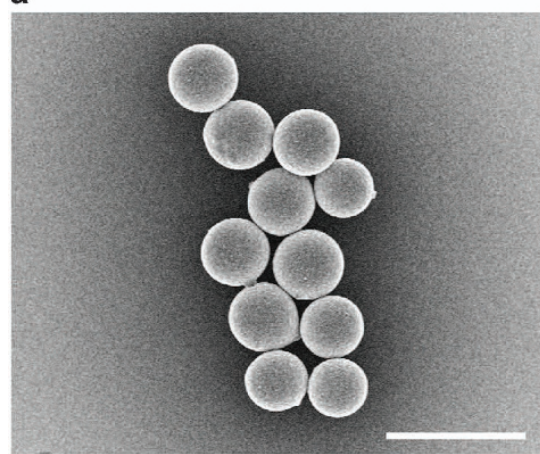

d

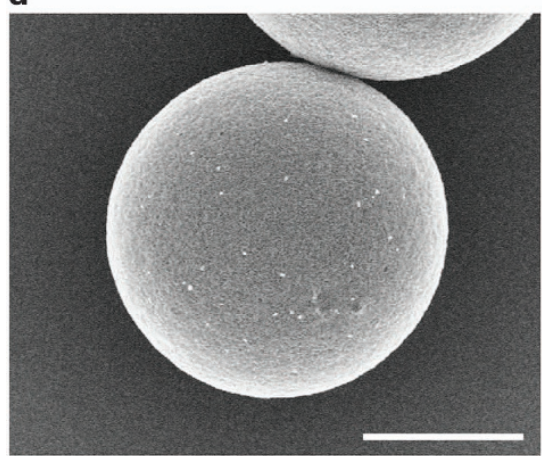

g

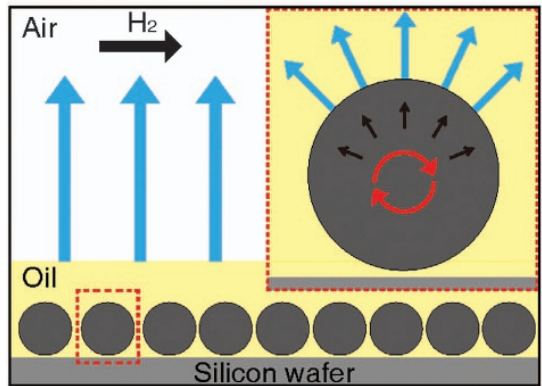

b

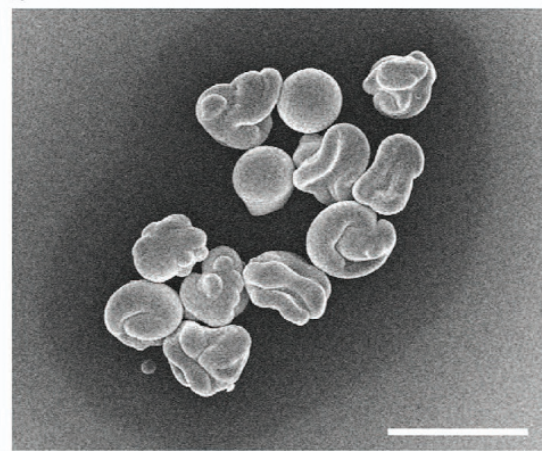

e

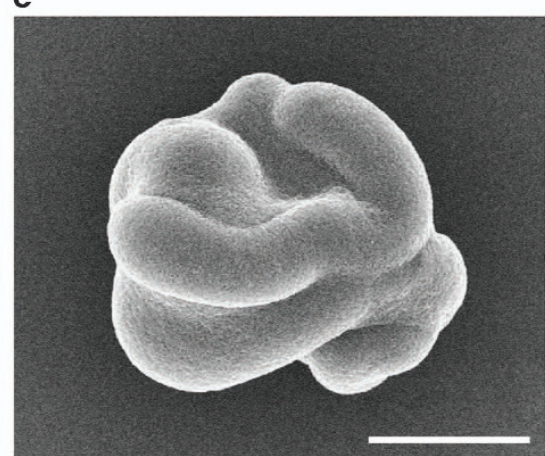

h

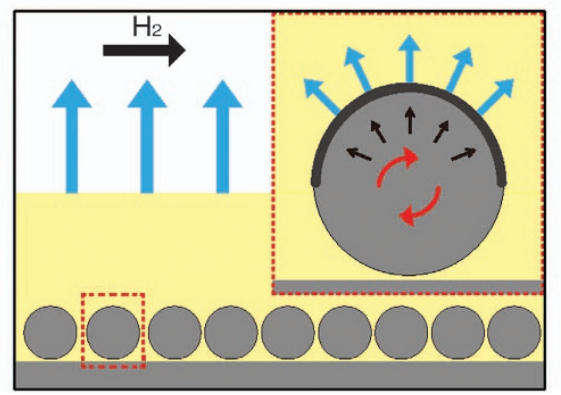

C

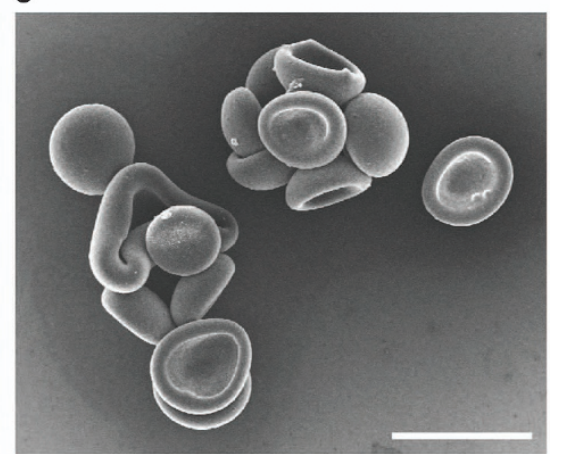

f

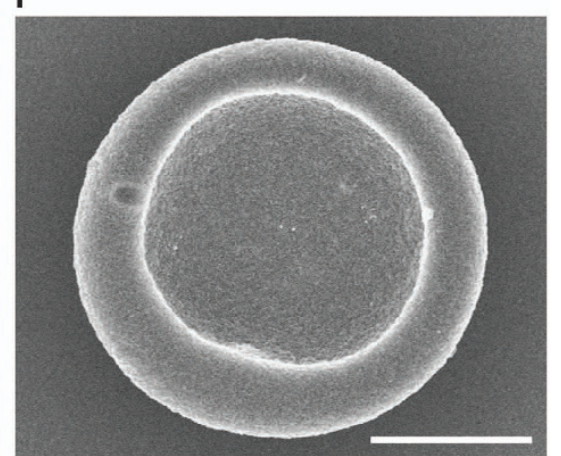

i

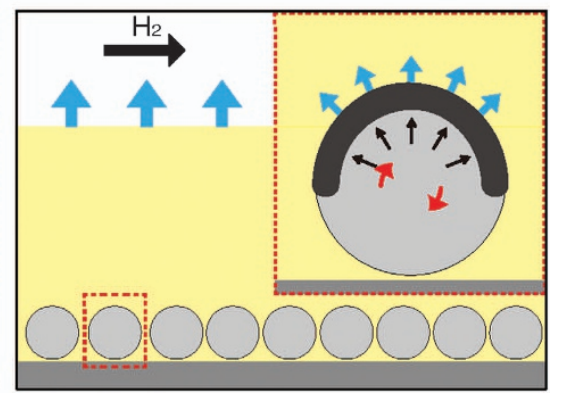

Figure 2 Diffusion-driven assembly of graphene oxides (GOs) in droplets. (a) Scanning electron microscopy (SEM) image of the spherical reduced graphene oxide ( $r G O)$ structure assembled under an oil layer thickness of $0.13 \mathrm{~mm}$. (b) SEM image of the twiddle rGO structure assembled under an oil layer thickness of $0.27 \mathrm{~mm}$. (c) SEM image of the hollow hemispherical rGO structure assembled under an oil layer thickness of $0.5 \mathrm{~mm}$. (d-f) Enlarged SEM images of a-c. (g-i) Schematic illustration of the assembly mechanism of GOs in droplets during the thermal diffusion process under the oil layer thicknesses of $0.13,0.27$ and $0.4 \mathrm{~mm}$. Blue arrows indicate the magnitude of the evaporation rate of water in the droplet to air. Insets of the enlarged red dotted box illustrate the circumstance of the droplet in details. Blue arrows of inlets indicate the magnitude of diffusion rate of water through the droplet interface. Dark arrows inside the droplet indicate the transfer of GOs from the center to the interface, and the circular red arrows show the magnitude of internal flux in the droplets. Scale bar (a-c): $20 \mu \mathrm{m}$ and $(\mathbf{d}-\mathbf{f}): 5 \mu \mathrm{m}$. 


\section{RESULTS AND DISCUSSION}

Our strategy for controlling the GO assembly in the droplet relies on tuning the diffusion rate of the aqueous solution by managing the oil quantity in the silicon wafer during the evaporation step. Figures $2 \mathrm{a}-\mathrm{c}$ show the 3D rGO assembly as a function of oil quantity and the corresponding enlarged images are displayed in Figures $2 \mathrm{~d}-\mathrm{f}$. In the process of the thermal treatment, water in the droplets diffused through the oil layer and then evaporated into the air. ${ }^{26}$ When water diffused upward through the water-oil interface, the GOs moved to the interface and tended to be accumulated. Note that the diffusion rate and accordingly the assembly behavior were significantly influenced by the thickness of the oil layer, although the solubility of water in hexadecane is minimal $\left(5.4 \times 10^{-3}\right.$ g per $100 \mathrm{~g}$ hexadecane). From Figure $2 \mathrm{a}-\mathrm{c}$, the oil layer became thicker as shown in the yellow color in Figures $2 \mathrm{~g}-\mathrm{i}$. Thus, the diffusion rate of an aqueous solution of droplets was slow, as the blue arrow indicates. ${ }^{26,27}$ Under $0.13-\mathrm{mm}$ thick oil layer, the water diffused into the oil phase relatively quickly with huge internal flux in the droplets (Figure $2 \mathrm{~g}$, inset). ${ }^{28}$ Such a vigorous internal flux rendered the GOs well dispersed inside the droplets, whereas the droplets were simultaneously shrunk by the

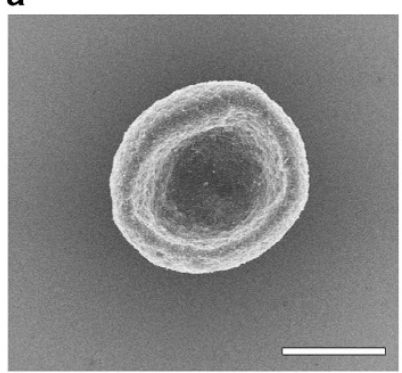

e

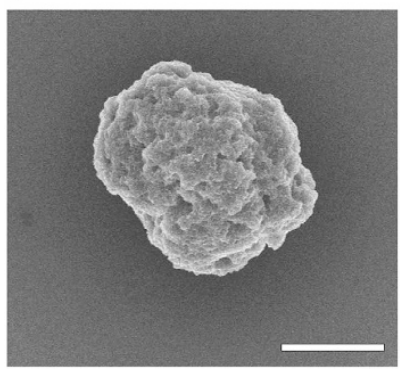

b

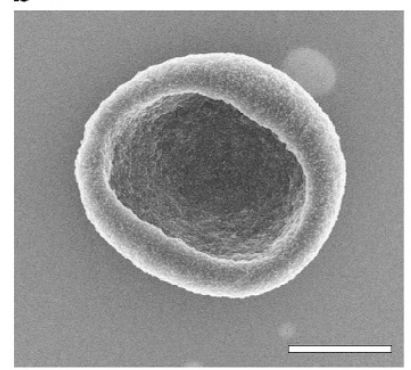

f

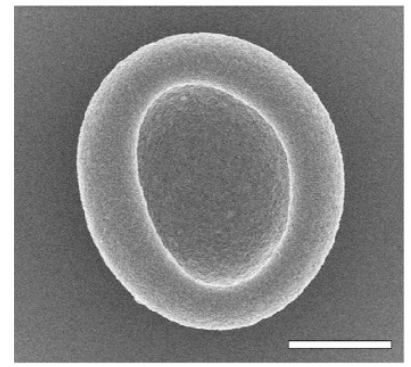

C

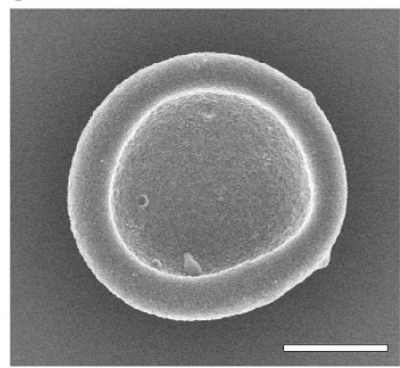

g

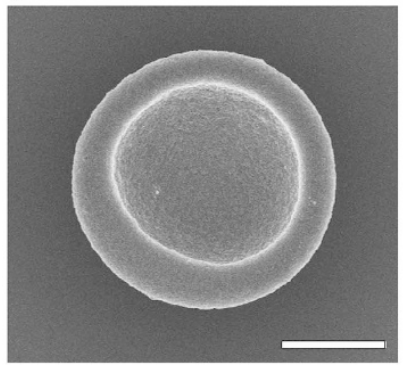

d

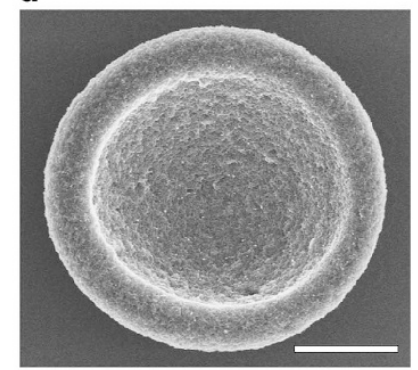

h

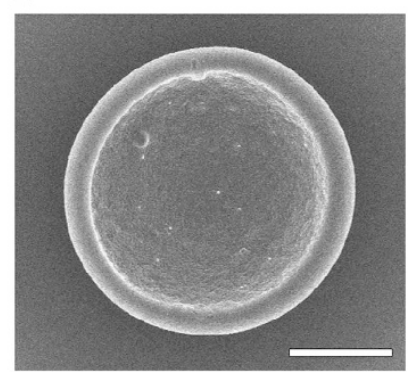

Figure 3 Tuning the size and thickness of the hollow hemispherical reduced graphene oxide (rGO) microparticles. (a-d) Scanning electron microscopy (SEM) images of the hollow hemispherical rGO microparticles with the GO concentration of $0.5,1,2$ and $4 \mathrm{mg} \mathrm{ml}^{-1}$, respectively. (e-h) SEM images of the rGO particles assembled in an oil layer of $0.4 \mathrm{~mm}$ in which the $\mathrm{pH}$ of the droplets was controlled at 1, 4, 7 and 10. Scale bar (a-h): $5 \mu \mathrm{m}$.

a

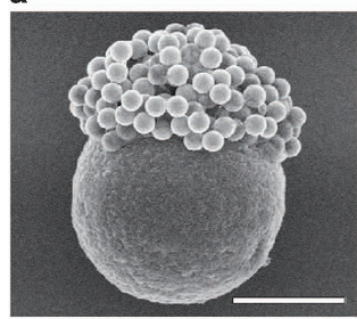

d

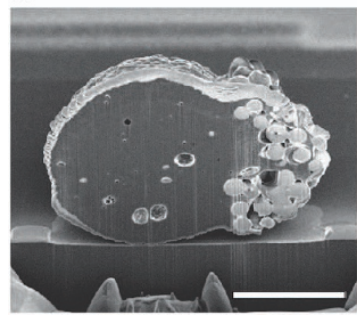

b

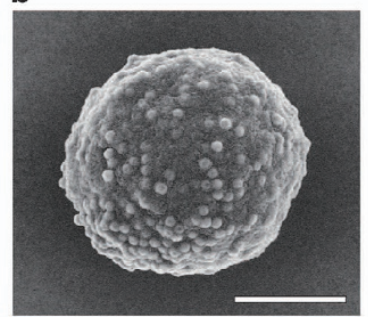

e

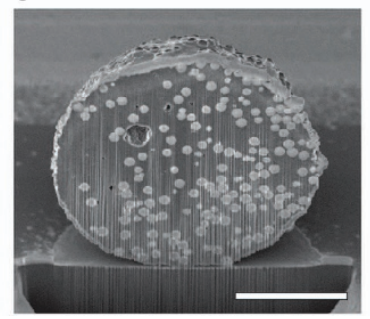

c

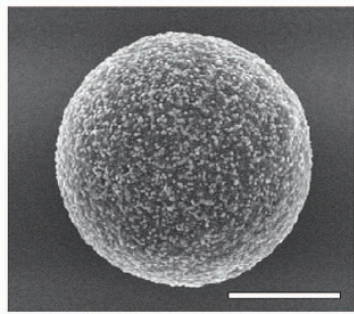

f

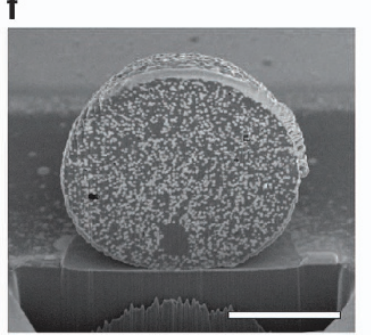

g

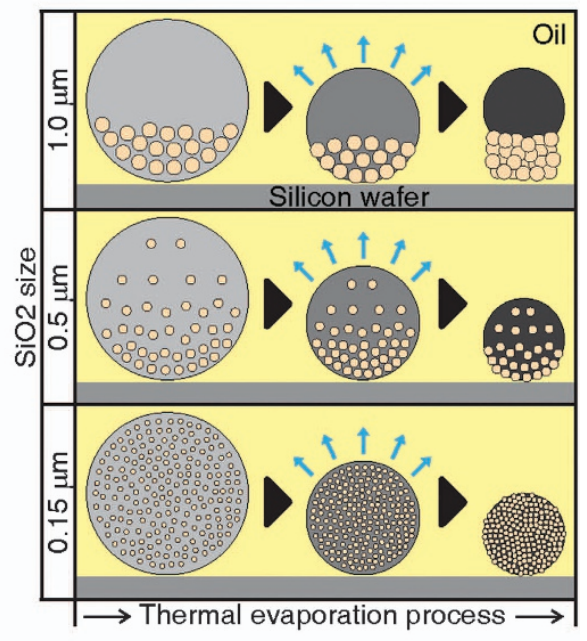

Figure 4 Spherical $\mathrm{rGO} \mathrm{SiO}_{2}$ composites with controlled internal bead distribution. (a-c) Scanning electron microscopy (SEM) images of the rGO-SiO 2 composites with the use of the silica beads of $1.0,0.5$ and $0.15 \mu \mathrm{m}$ diameter. (d-f) Cross-sectional SEM images of (a-c) by using a focused ion beam (FIB) instrument. (g) Schematic illustration of the assembly mechanism of the spherical $\mathrm{rGO}^{-\mathrm{SiO}_{2}}$ depending on the bead size. Scale bar (a-f): $5 \mu \mathrm{m}$. 
loss of water. During this process, Laplace pressure, which compresses the droplets along the axis perpendicular to the droplet interface, was applied on the droplets. The GOs were gathered and assembled in the center of the droplets, forming a spherical structure (Figures $2 \mathrm{~d}$ and g). With a 0.27 -mm-thick oil layer, the diffusion rate decreased to induce a moderate internal flux that led to the accumulation of GOs at the top of the water-oil interface (Figure 2b). As the bottom of the droplets was blocked by the silicon wafer, the water of the droplets mainly diffused through the top side of the droplet, thereby making the GOs aggregate on the top portion of the droplets to form a curved GO film (Figure 2h, inset). ${ }^{28,29}$ At a certain point, the GO films collapsed and compressed because of Laplace pressure, resulting in the twiddle morphology (Figures $2 \mathrm{~b}$ and e). When a relatively large amount of oil was used (a 0.4-mm-thick oil layer), the water diffusion was much slower and a weak internal flux was induced (Figure 2i, inset). Thus, the GO sheets would be dominantly accumulated at the top interface and produced a thick GO film that was enough to endure

a

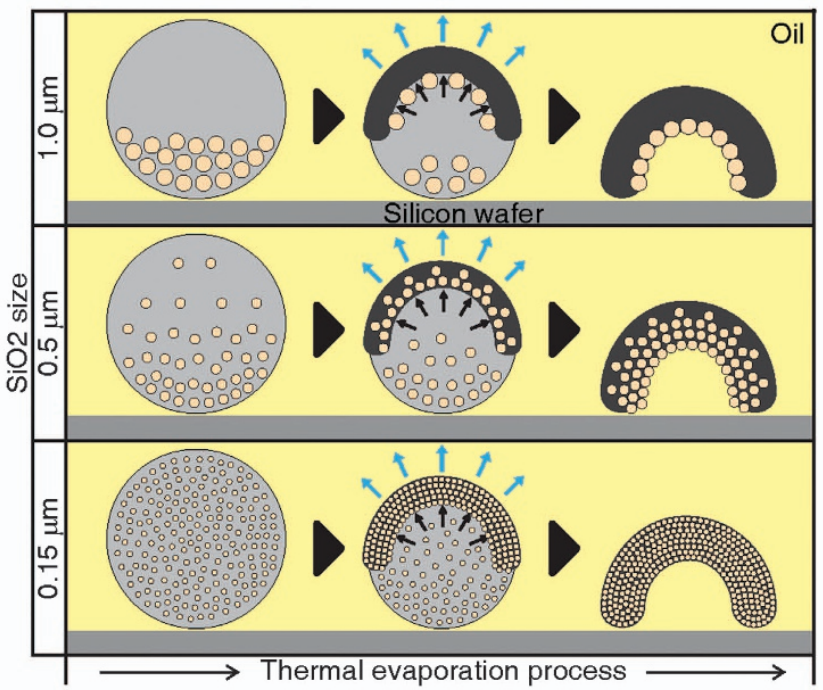

C

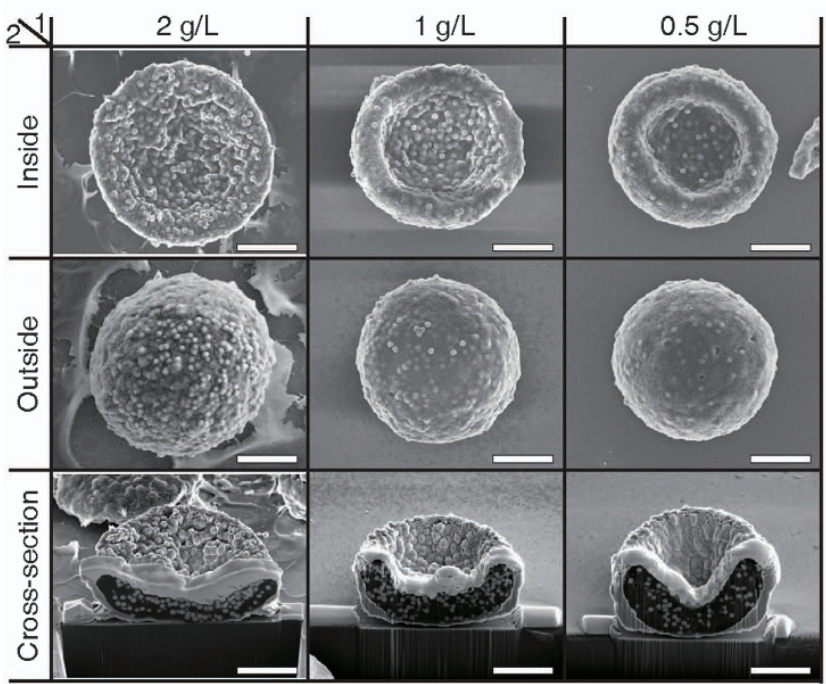

the Laplace pressure, forming hollow hemispherical structures (Figures $2 \mathrm{c}$ and $\mathrm{f}$ ). However, when the oil layer increased to $0.53 \mathrm{~mm}$, the diffusion rate was so slow that the droplets burst before the GOs were assembled, leaving no GO particles on the substrate. These results imply that the oil layer thickness could simply but significantly control the diffusion rate of the aqueous solution of the droplets, so that the GO assembling in the droplets could be manipulated to produce diverse morphologies of $3 \mathrm{D}$ rGO. In particular, the anisotropic diffusion of GO drastically changed the structure of rGO from sphere to twiddle sphere to hollow hemisphere. The hydrophobic surface treatment on the silicon wafer allowed the droplets to be lifted from the surface, so that the 3D graphene structure could be recovered intact.

Among the produced 3D rGO, we were particularly interested in the hollow hemisphere because of its distinct shape. First of all, we controlled the size as well as the thickness of the shell. For the size control, the concentration of the GO in the droplets was adjusted at

b

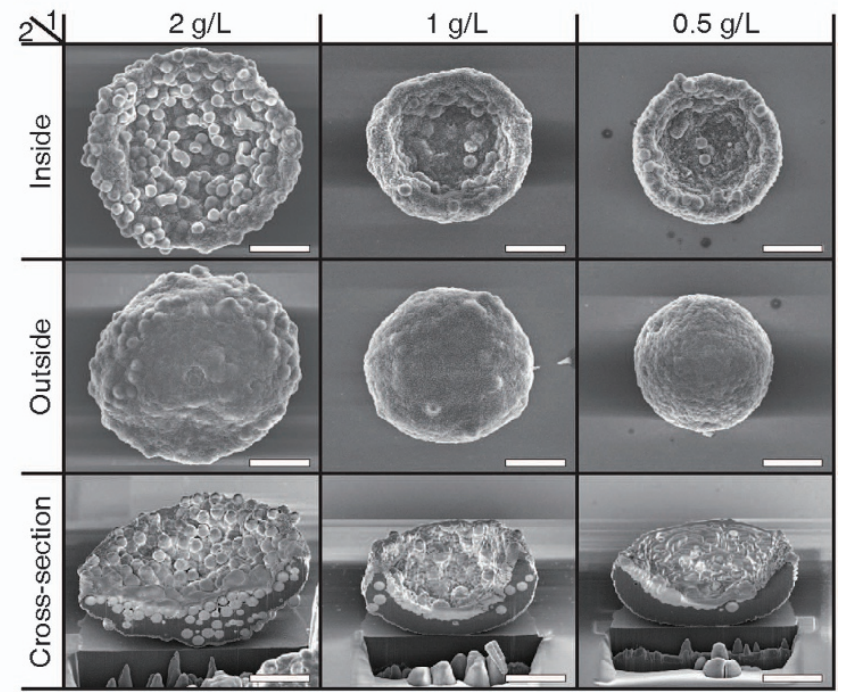

d

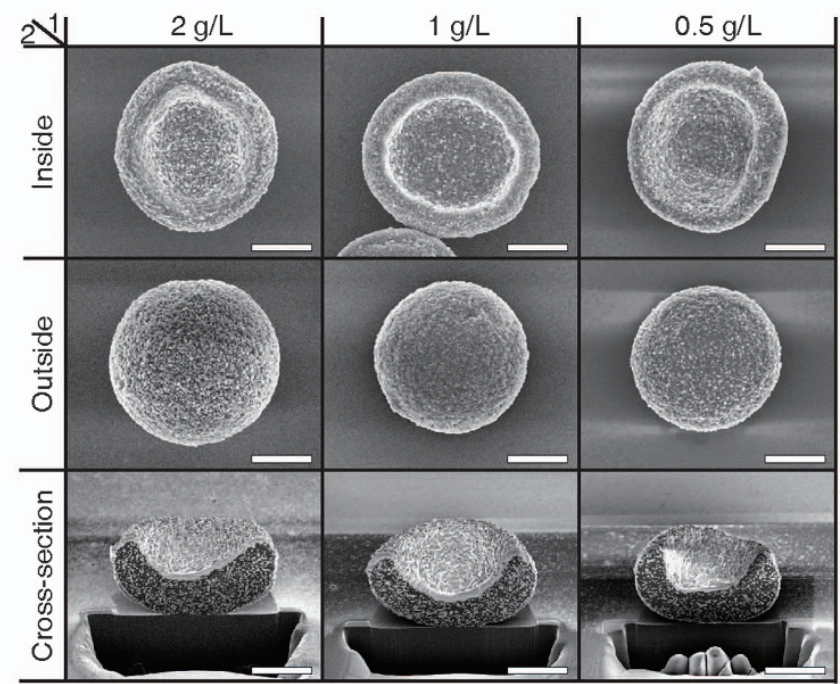

Figure 5 Hollow hemispherical rGO-SiO 2 composites with the controlled internal bead distribution. (a) Schematic illustration of the assembly mechanism of the hollow hemispherical rGO-SiO ${ }_{2}$. (b-d) Scanning electron microscopy (SEM) images of the hollow hemispherical rGO-SiO 2 with variation of the bead size $\left(1.0 \mu \mathrm{m}\right.$ for (b), $0.5 \mu \mathrm{m}$ for (c) and $0.15 \mu \mathrm{m}$ for (d), respectively) and concentration (2, 1 and $\left.0.5 \mathrm{gl}^{-1}\right)$. On the table label, 1 and 2 represent the concentration of silica beads and the view aspects (inside, outside, cross-section), respectively. Scale bar (b-d): $5 \mu \mathrm{m}$. 
$0.5,1,2$ and $4 \mathrm{mg} \mathrm{ml}^{-1}$. Whereas the hollow hemispherical structure was retained, the diameter and shell thickness of the hemisphere gradually increased in proportion to the GO concentration (Figures 3a-d). However, the low concentration at $0.1 \mathrm{mg} \mathrm{ml}^{-1}$ produced a squashed lump (Supplementary Figure S1a). It seems that such a low concentration of GO initially generated a thin film that was then completely compressed by Laplace pressure. Thus, at least a sufficient amount of GO is necessary to endure the Laplace pressure to maintain the hollow hemispherical shape. On the other hand, if a high concentration of $6 \mathrm{mg} \mathrm{ml}^{-1}$ was used, the GO-filled hemisphere was synthesized (Supplementary Figure S1b). In that case, the GOs would be continuously accumulated inside the top film during the water diffusion. These results suggest that an optimal range of the GO concentration was required to produce the hollow hemispherical rGO.

In addition to the size control, we could also tune the shell thickness. As the GO was abundant in the negative oxygenous functional groups, we adjusted the $\mathrm{pH}$ of the droplets to manipulate the degree of $\mathrm{GO}$ aggregation. At low $\mathrm{pH}$, the GOs tend to be aggregated, whereas they are well dispersed at high $\mathrm{pH}^{30}$ It was reported that the GO sheets were dominantly arranged with an edgeto-edge connection at low $\mathrm{pH}$, whereas the face-to-face overlap was preferred at high $\mathrm{pH} .{ }^{19,20,31}$ Considering such a $\mathrm{pH}$-dependent GO assembly behavior, we controlled the $\mathrm{pH}$ in the droplets to tune the thickness of the hollow hemispherical structure (Figures $3 \mathrm{e}-\mathrm{h}$ ). Acidic conditions $(\mathrm{pH} 1)$ led to strong attraction among the GO sheets with wrinkled edges, resulting in a lump with rough surface. However, as the $\mathrm{pH}$ value increased from 4 to 10 , the repulsion between the GO sheets became significant and assembled in a layer-by-layer manner, so the shell thickness was gradually thinner. The thickness of the hollow hemisphere was reduced to $2.63 \pm 0.09,2.0 \pm 0.10$ and $1.39 \pm 0.04 \mu \mathrm{m}$ at $\mathrm{pH} \mathrm{4,7}$ and 10, respectively. These results imply that we could adjust the size and the thickness of the hollow hemisphere by controlling the GO concentration and $\mathrm{pH}$ inside the micro-droplet.

We further investigated the fabrication of the 3D rGO nanocomposites with silica beads. For this purpose, we utilized the convective microscale flow inside the droplets to embed silica beads in the rGO architecture. Under the synthetic conditions for spherical shapes, silica beads with different sizes (diameter of $1.0,0.5$ and $0.15 \mu \mathrm{m}$ with concentration of $1 \mathrm{gl}^{-1}$ ) were added, producing a distinct internal distribution of the silica beads in the GO matrix depending on the size of the silica beads (Figures $4 \mathrm{a}-\mathrm{f}$ ). The use of the $1.0 \mu \mathrm{m}$ silica beads resulted in the Janus structure of rGO with silica beads (Figures $4 \mathrm{a}$ and $\mathrm{d}$ ). In the case of $0.5 \mu \mathrm{m}$ silica beads, the bead density became less from right to left in the rGO sphere (Figures $4 \mathrm{~b}$ and e), whereas the $0.15 \mu \mathrm{m}$ silica beads were uniformly distributed inside the rGO sphere (Figures $4 \mathrm{c}$ and $\mathrm{f}$ ). It seems that the flux inside the micro-droplets, which sufficiently induced the GO assembly to the sphere structure, was not enough to transport microscale silica beads such as the nanosized GOs. Such a size-dependent distribution of the silica beads in the droplets can be explained by a modified sedimentation equilibrium that describes vertical density gradient of a Brownian particle $^{32}$ (Supplementary Information S2),

$$
n=n_{0} \exp \frac{-4 \pi r^{3} g\left(\rho-\rho^{\prime}\right) h}{3 k T+18 \eta v / r}
$$

where $n_{0}$ and $n$ are the density of particles at height $h=0$ and $h, r$ is the radius of particles, $g$ is gravitational acceleration and $\rho$ and $\rho^{\prime}$ are the density of the beads and the fluid. $k$ and $T$ are the Boltzmann constant and temperature, $\eta$ and $v$ are viscosity and flow velocity, respectively. The ratio of $n_{0} / n$ is proportional to the radius of the particle. Thus, if the size of the silica beads was larger, the number of the beads at the bottom of the droplets would increase. In the opposite case, the smaller beads would be equally distributed along the height in the droplets. Figure $4 \mathrm{~g}$ displays the schematics of the silica bead distribution depending on size. In the case of $1.0 \mu \mathrm{m}$ silica beads, the gravitational force would be greater than the dispersive force that is the combination of the internal flux force with the buoyance force, and hence the silica beads sunk. In the case of $0.5 \mu \mathrm{m}$ silica beads, the dispersive force is more dominant owing to the reduced weight of beads, forming a density gradient in the internal structure. When $0.15 \mu \mathrm{m}$ sized beads are used, a relatively large dispersive force is exerted, distributing the beads uniformly inside the rGO microparticles. The results clearly show that the distribution of the silica beads in the rGO sphere could be controlled by their size, whereas the nano- a

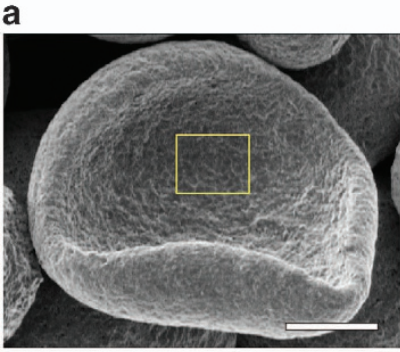

e

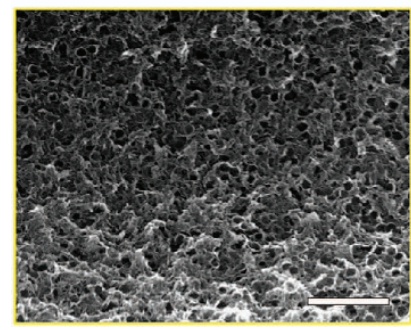

b

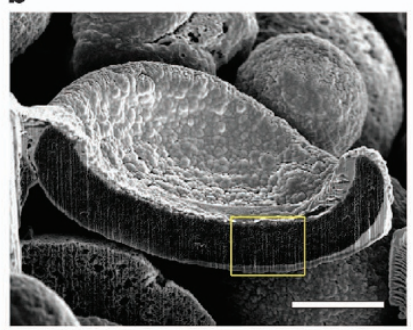

f

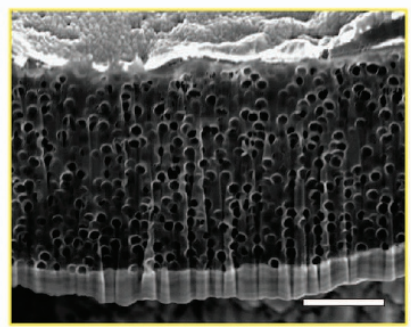

c

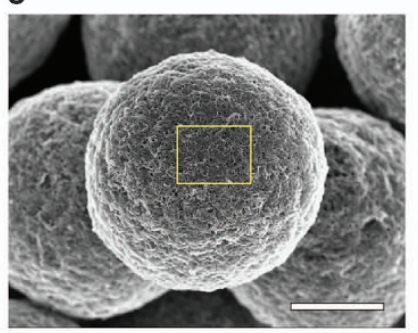

g

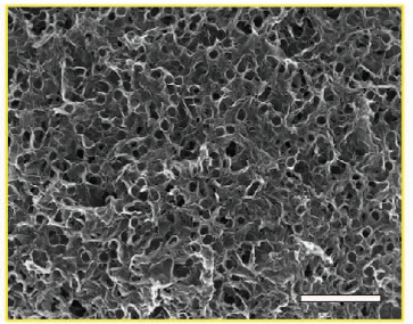

d

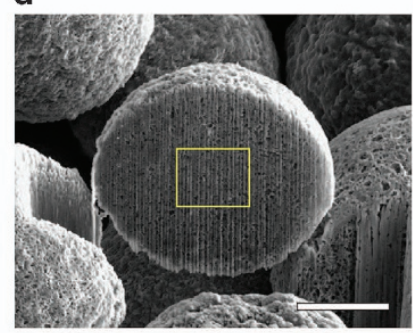

$\mathbf{h}$

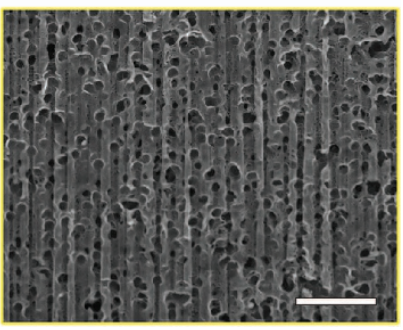

Figure 6 Porous reduced graphene oxide ( $\mathrm{rGO}$ ) microstructures produced by etching silica beads. Scanning electron microscopy (SEM) images of (a) the inner curvature and (b) the cross-section of the porous hollow hemispherical rGO. SEM images of (c) the surface and (d) the cross-section of the porous spherical rGO. (e-h) Enlarged SEM images of (a-d). Scale bar (a-d): $5 \mu \mathrm{m}$ and (e-h): $1 \mu \mathrm{m}$. 
sized GO sheets were aggregated as microparticles because of the high internal flux as described in Figure 2g.

Such a tendency was also found in the hollow hemispherical structure. As shown in the schematics of Figure 5a, the silica beads of $1.0 \mu \mathrm{m}$ would settle down at the bottom of the droplets and are much more slowly transported upward than the GO sheets, and hence the beads would be embedded inside the concaved rGO. The scanning electron microscopy images of Figure $5 \mathrm{~b}$ confirm that the beads were located only inside the shell. As the bead concentration increased from 0.5 to $2 \mathrm{gl}^{-1}$, the silica beads in the concave surface were more densely packed. The use of the $0.5 \mu \mathrm{m}$ silica beads would result in a bead gradient in the rGO shell because of the convective internal flux that carried some of the beads upward (Figure $5 \mathrm{a}$, the middle panel). We could observe more beads inside the shell than those outside the shell, and the cross-sectional image displayed more densely packed beads in the inner part of the shell, whereas the convex side was mainly covered by rGO (Figure 5c). Finally, in case of the $0.15 \mu \mathrm{m}$ silica beads, the movement of the GO and the bead toward the top of the droplets is comparable, and hence the beads would be uniformly distributed in the hollow hemispherical rGO (Figure 5a, the bottom panel). Figure $5 \mathrm{~d}$ clearly shows the uniform distribution of the $0.15 \mu \mathrm{m}$ beads within the hollow hemispherical rGO shell. The variation of bead concentration rarely influenced the bead distribution, but the curvature of the hollow hemispherical rGO was reduced as the bead concentration increased.

As-synthesized rGO-SiO 2 composites can be further modified by dissolving silica beads in hydrofluoric acid, producing porous structure. As the bead size and distribution can be controlled as described above, the complicated 3D rGO with tunable pore sizes and spatial distributions could be achieved. As a model, spherical and hollow hemispherical rGO-SiO 2 composites, which were synthesized with $0.15 \mu \mathrm{m}$ diameter silica beads and $2.6 \mathrm{gl}^{-1}$ concentration, were chosen and the wet etching process was performed to remove the beads. Figures $6 \mathrm{a}-\mathrm{h}$ show the scanning electron microscopy images of the porous spherical and porous hollow hemispherical rGO structures. As we utilized a high concentration of the silica beads, we could observe the pores throughout the $3 \mathrm{D}$ rGO. Such a porous graphene material has significant potential to be used in the fields of energy storage, catalysis, $\mathrm{CO}_{2}$ capture, adsorption and separation. 5,13,33,34

Recent reports presented the unique surface plasmonic properties of graphene, and graphene is regarded as the ideal material for the nextgeneration photonic device. ${ }^{35}$ Because the plasmonic phenomenon of graphene depends on the thickness as well as the shape, we are particularly interested in the optical plasmonic properties of the $3 \mathrm{D}$ porous hollow hemispherical graphene structure and investigated its plasmonic effect spatially and spectrally by using a monochromated EELS in an aberration-corrected STEM. Experimental details are described in the Supplementary Information S3. We targeted the vacant shell for the EELS mapping (Figures $7 \mathrm{~b}-\mathrm{e}$ ), and the EELS spectrum shows a $\pi$ plasmon peak at $5.4 \mathrm{eV}$ and a $\pi+\sigma$ plasmon peak at $24 \mathrm{eV}$ that were similar to those of multilayer graphene ( $>5$ layers) because of the stacked rGOs (Figure 7a) ${ }^{36,37}$. Theoretical calculation for graphene plasmon and its correlation with the sample thickness have revealed that $\pi$ plasmon at $4.5 \mathrm{eV}$ and $\pi+\sigma$ plasmon at $15 \mathrm{eV}$ are characteristic of the surface plasmon, whereas $\pi+\sigma$ plasmon at $25 \mathrm{eV}$ is indicative of the bulk plasmon. ${ }^{36-38}$

Herein, we utilized the EELS mapping with an energy loss response at 5.4, 15 and $24 \mathrm{eV}$ to comprehensively understand the plasmonic properties of the vacant graphene ball (Supplementary Figure S2b, red box). The targeted 3D graphene structure allowed us to obtain the EELS imaging on both the thin thickness at the center (the inner part of

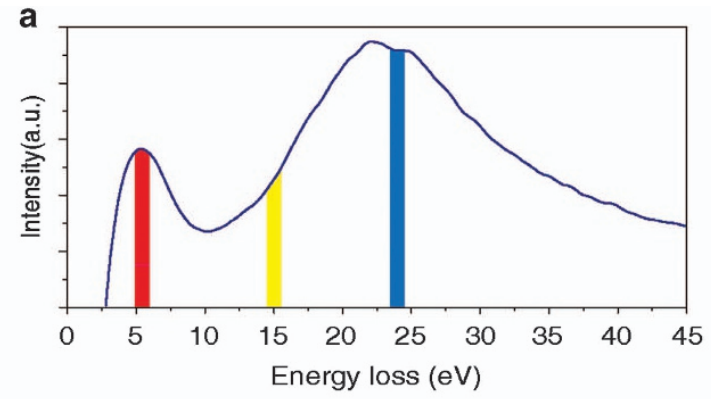

b

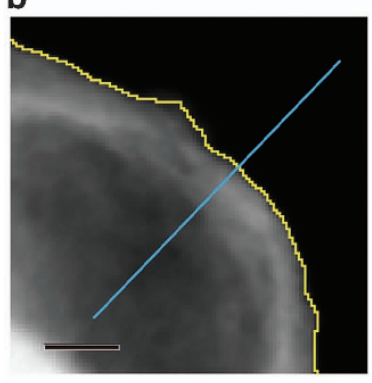

d

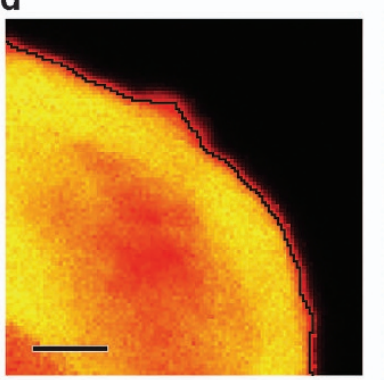

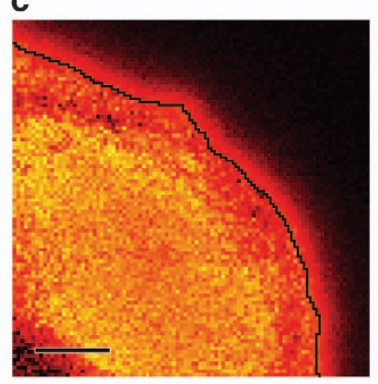

e

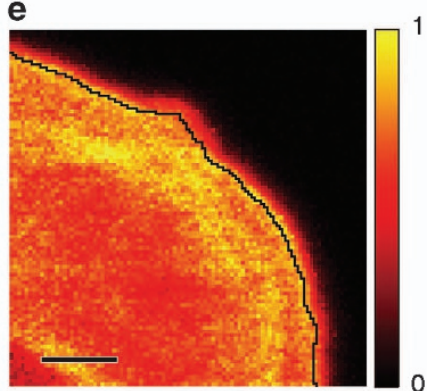

Figure 7 Optical plasmon mapping of the porous reduced graphene oxide (rGO) structures with scanning transmission electron microscopy-electron energy loss spectroscopy (STEM-EELS). (a) An EELS spectrum acquired by focusing on the vacant graphene ball (the red box of Supplementary Figure S2b). Red, yellow and blue bands indicate an energy loss range of 4.9-5.9, 14.5-15.5 and 23.5-24.5 eV, respectively. (b) A high-angle annular dark-field (HAADF) image of the vacant pore at the edge area of the porous hollow hemispherical rGO (an enlarged HAADF image of the red box of Supplementary Figure S2b). (c) A plasmon map of 4.9-5.9 eV. (d) A plasmon map of 23.5-24.5 eV. (e) A plasmon map of $14.5-15.5 \mathrm{eV}$. Scale bar (b-e): $10 \mathrm{~nm}$.

Figure 7b) and the thick thickness at the edge (the close boundary of the yellow line in Figure $7 \mathrm{~b}$ ) (see the schematics in Supplementary Figures S2c and d). We also noted the yellow boundary line between the surface and the vacuum to investigate the distance of the aloof excitation that suggests the existence of surface plasmons. ${ }^{39-41}$ The EELS imaging using the signal at $5.4 \mathrm{eV}$ shows a high plasmonic intensity at the center, a low intensity at the edge and a long aloof plasmon excitation, confirming that $5.4 \mathrm{eV}$ is a surface plasmon (Figure $7 \mathrm{c}$ ). At $24 \mathrm{eV}$ of $\pi+\sigma$ plasmon, a high plasmonic intensity on the edge was displayed with a short aloof excitation, suggesting that $24 \mathrm{eV}$ is a bulk plasmon (Figure $7 \mathrm{~d}$ ). Although the $\pi+\sigma$ plasmon peak at $15 \mathrm{eV}$ was not clear because of the overlap of the broad $24 \mathrm{eV}$ peak in Figure 7a, we produced the EELS image of the low-loss response at $15 \mathrm{eV}$ to check the intensity distribution near the surface (Figure 7e). In this case, a high intensity of plasmon was detected at the edge part (bulk plasmon) and, interestingly, a relatively long aloof excitation distinctly coexisted, meaning the presence of the surface 
plasmon characteristic. Comparison of the distance and intensity of the aloof excitation is described in the Supplementary Information S4.

In conclusion, we demonstrated a variety of the 3D rGO architectures by controlling the diffusion rate of the aqueous solution in the micro-droplet reactors. The GO assembly pattern was determined by manipulating the degree of the internal flux in the droplets, so the sphere, twiddle and hollow hemisphere rGO could be obtained. In particular, the size and the thickness of the hollow hemisphere rGO were tuned by the concentration of the GO and the $\mathrm{pH}$ in the droplets. In addition, their composites with the silica beads could be prepared with controllable internal distribution, and through the wet etching process, the porous $3 \mathrm{D}$ rGO was fabricated. Noticeably, the porous hemispherical rGO structure shows the local surface plasmon as well as the bulk plasmon that can be useful in the fields of surface-enhanced Raman spectroscopy, photocatalysis, photothermal converter and energy storage.

\section{CONFLICT OF INTEREST}

The authors declare no conflict of interest.

\section{ACKNOWLEDGEMENTS}

This research was supported by Basic Science Research Program through the National Research Foundation of Korea (NRF) funded by the Ministry of Science, ICT \& Future Planning (No. 2014R1A5A1009799), and C1 Gas Refinery Program through the National Research Foundation of Korea (NRF) funded by the Ministry of Science, ICT \& Future Planning (2015M3D3A1A01064882).

1 Geim, A. K. Graphene: status and prospects. Science 324, 1530-1535 (2009).

2 Lee, C., Wei, X., Kysar, J. W. \& Hone, J. Measurement of the elastic properties and intrinsic strength of monolayer graphene. Science 321, 385-388 (2008).

3 Liu, F., Jang, M. H., Ha, H. D., Kim, J. H., Cho, Y. H. \& Seo, T. S. Facile synthetic method for pristine graphene quantum dots and graphene oxide quantum dots: origin of blue and green luminescence. Adv. Mater. 25, 3657-3662 (2013).

4 Zou, J. \& Kim, F. Diffusion driven layer-by-layer assembly of graphene oxide nanosheets into porous three-dimensional macrostructures. Nat. Commun. 5, 5254 (2014).

5 Jiang, L. \& Fan, Z. Design of advanced porous graphene materials: from graphene nanomesh to 3D architectures. Nanoscale 6, 1922-1945 (2014).

6 Peplow, M. Graphene booms in factories but lacks a killer app. Nature 522, 268 (2015).

7 Chabot, V., Higgins, D., Yu, A., Xiao, X., Chen, Z. \& Zhang, J. A review of graphene and graphene oxide sponge: material synthesis and applications to energy and the environment. Energy Environ. Sci. 7, 1564-1596 (2014).

8 Liu, F., Piao, Y., Choi, K. S. \& Seo, T. S. Fabrication of free-standing graphene composite films as electrochemical biosensors. Carbon 50, 123-133 (2012).

9 Park, S., Kim, H.-K., Yoon, S., Lee, C., Ahn, D., Lee, S.-I., Roh, K. C. \& Kim, K.-B. Spray-assisted deep-frying process for the in situ spherical assembly of graphene for energy-storage devices. Chem. Mater. 27, 457-465 (2014).

10 Han, D. J., Jung, J. H., Choi, J. S., Kim, Y. T. \& Seo, T. S. Synthesis of a 3D graphite microball using a microfluidic droplet generator and its polymer composite with core-shell structure. Lab Chip. 13, 4006-4010 (2013).

11 Smith, A. J., Chang, Y.-H., Raidongia, K., Chen, T.-Y., Li, L.-J. \& Huang, J. Molybdenum sulfide supported on crumpled graphene balls for electrocatalytic hydrogen production. Adv. Energy Mater. 4, 1400398 (2014).

12 Luo, J., Gao, J., Wang, A. \& Huang, J. Bulk nanostructured materials based on two-dimensional building blocks: a roadmap. ACS Nano 9, 9432-9436 (2015).

13 Raccichini, R., Varzi, A., Passerini, S. \& Scrosati, B. The role of graphene for electrochemical energy storage. Nat. Mater. 14, 271-279 (2015).

14 Cao, X., Yin, Z. \& Zhang, H. Three-dimensional graphene materials: preparation, structures and application in supercapacitors. Energy Environ. Sci. 7, 1850-1865 (2014).

15 Dreyer, D. R., Park, S., Bielawski, C. W. \& Ruoff, R. S. The chemistry of graphene oxide. Chem. Soc. Rev. 39, 228-240 (2010).

16 Stankovich, S., Dikin, D. A., Piner, R. D., Kohlhaas, K. A., Kleinhammes, A., Jia, Y., Wu, Y., Nguyen, S. T. \& Ruoff, R. S. Synthesis of graphene-based nanosheets via chemical reduction of exfoliated graphite oxide. Carbon 45, 1558-1565 (2007)
17 Cote, L. J., Kim, F. \& Huang, J. Langmuir-Blodgett assembly of graphite oxide single layers. J. Am. Chem. Soc. 131, 1043-1049 (2009).

$18 \mathrm{Kim}, \mathrm{F} .$, Laura, J. \& Huang, J. Graphene oxide: surface activity and two-dimensional assembly. Adv. Mater. 22, 1954-1958 (2010).

19 Kim, J., Cote, L. J., Kim, F., Yuan, W. \& Shull, K. R. Graphene oxide sheets at interfaces. J. Am. Chem. Soc. 132, 8180-8186 (2010).

20 Cote, L. J., Kim, J., Zhang, Z., Sun, C. \& Huang, J. Tunable assembly of graphene oxide surfactant sheets: wrinkles, overlaps and impacts on thin film properties. Soft Matter $\mathbf{6}$, 6096-6101 (2010).

21 Wei, L., Chen, F., Wang, H., Zeng, T. H., Wang, Q. \& Chen, Y. Acetone-induced graphene oxide film formation at the water-air interface. Chem. Asian J. 8, 437-443 (2013).

22 Luo, J., Jang, H. D., Sun, T., Xiao, L., He, Z., Katsoulidis, A. P., Kanatzidis, M. G., Gibson, J. M. \& Huang, J. Compression and aggregation-resistant particles of crumpled soft sheets. ACS Nano 5, 8943-8949 (2011).

23 Xu, S., Nie, Z., Seo, M., Lewis, P., Kumacheva, E., Stone, H. A., Garstecki, P., Weibel, D. B., Gitlin, I. \& Whitesides, G. M. Generation of monodisperse particles by using microfluidics: control over size, shape, and composition. Angew. Chem. Int. Ed. 44, 724-728 (2005).

24 Song, H., Chen, D. L. \& Ismagilov, R. F. Reactions in droplets in microfluidic channels. Angew. Chem. Int. Ed. 45, 7336-7356 (2006).

25 Fang, A., Gaillard, C. \& Douliez, J. P. Template-free formation of monodisperse doughnut-shaped silica microparticles by droplet-based microfluidics. Chem. Mater. 23, 4660-4662 (2011).

26 Aranberri, I., Beverley, K. J., Binks, B. P., Clint, J. H. \& Fletcher, P. D. I. How do emulsions evaporate? Langmuir 18, 3471-3475 (2002).

27 Schatzberg, P. Solubilities of water in several normal alkanes from C7 to C16. J. Phys. Chem. 67, 776-779 (1963).

28 Chang, S. T. \& Velev, O. D. Evaporation-induced particle microseparations inside droplets floating on a chip. Langmuir 22, 1459-1468 (2006).

29 Millman, J. R., Bhatt, K. H., Prevo, B. G. \& Velev, O. D. Anisotropic particle synthesis in dielectrophoretically controlled microdroplet reactors. Nat. Mater. 4, 98-102 (2004).

30 Shih, C.-J., Lin, S., Sharma, R., Strano, M. S. \& Blankschtein, D. Understanding the $\mathrm{pH}$-dependent behavior of graphene oxide aqueous solutions: a comparative experimental and molecular dynamics simulation study. Langmuir 28, 235-241 (2012).

31 Cote, L. J., Kim, J., Tung, V. C., Luo, J., Kim, F. \& Huang, J. Graphene oxide as surfactant sheets. Pure Appl. Chem. 83, 95-110 (2010).

32 Perrin, J. Atoms (Ox Bow Press: Woodbridge, CT, USA, 1990)

33 Liu, J., Wickramaratne, N. P., Qiao, S. Z. \& Jaroniec, M. Molecular-based design and emerging applications of nanoporous carbon spheres. Nat. Mater. 14, 763-774 (2015)

34 Knox, J. H., Kaur, B. \& Millward, G. R. Structure and performance of porous graphitic carbon in liquid chromatography. J. Chromatogr. 352, 3-25 (1986).

35 Jablan, M., Soljacic, M. \& Buljan, H. Plasmons in graphene: fundamental properties and potential applications. Proc. IEEE 101, 1689-1704 (2013).

36 Gass, M. H., Bangert, U., Bleloch, A. L., Wang, P., Nair, R. R. \& Geim, A. K. Freestanding graphene at atomic resolution. Nat. Nanotechnol. 3, 676-681 (2008).

37 Eberlein, T., Bangert, U., Nair, R. R., Jones, R., Gass, M., Bleloch, A. L., Novoselov, K. S., Geim, A. K. \& Briddon, P. R. Plasmon spectroscopy of free-standing graphene films. Phys. Rev. B 77, 233406 (2008).

38 Carbone, F., Barwick, B., Kwon, O., Park, H. S., Baskin, J. S. \& Zewail, A. H. EELS femtosecond resolved in 4D ultrafast electron microscopy. Chem. Phys. Lett. 468, 107-111 (2009).

39 Browning, N. D., Arslan, I., Erni, R. \& Reed, B.W. in Scanning Transmission Electron Microscopy (eds Pennycook, S. J. \& Nellist, P. D.) 659-688 (Springer: New York, NY, USA, 2011).

40 Garciía De Abajo, F. J. Multiple excitation of confined graphene plasmons by single free electrons. ACS Nano 7, 11409-11419 (2013).

41 Ooi, K. J. A., Koh, W. S., Chu, H. S., Tan, D. T. H. \& Ang, L. K. Efficiencies of aloofscattered electron beam excitation of metal and graphene plasmons. IEEE Trans. Plasma Sci. 43, 951-956 (2011).

This work is licensed under a Creative Commons Attribution 4.0 International License. The images or other third party material in this article are included in the article's Creative Commons license, unless indicated otherwise in the credit line; if the material is not included under the Creative Commons license, users will need to obtain permission from the license holder to reproduce the material. To view a copy of this license, visit http:// creativecommons.org/licenses/by/4.0/

(C) The Author(s) 2016

Supplementary Information accompanies the paper on the NPG Asia Materials website (http://www.nature.com/am) 